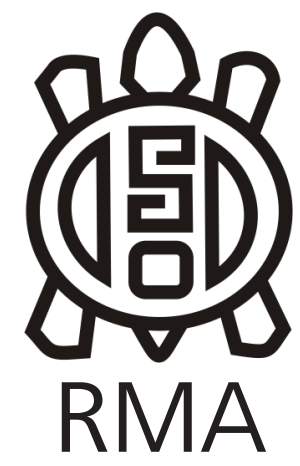

Dossier

\section{Análisis funcional de base microscópica aplicado a materias primas de la provincia de Entre Ríos: identificación y caracterización de rastros de uso experimentales}

\author{
Microscopic functional analysis applied to lithic raw materials from \\ Entre Ríos province: identification and characterization of experimental
}

use wear

\begin{abstract}
*FCNyM - División Arqueología del Museo de La Plata, Facultad de Ciencias Naturales y Museo, U.N.L.P mleipus@fcnym.unlp.edu.ar "*CONICET - División Arqueología del Museo de La Plata, Facultad de Ciencias Naturales y Museo, U.N.L.P eapolinaire@fcnym.unlp.edu.ar
\end{abstract}

\begin{abstract}
Resumen
El objetivo de este trabajo es presentar los resultados del programa experimental de rastros de uso aplicado a materias primas líticas procedentes de diferentes afloramientos del territorio entrerriano que son detectadas en los contextos arqueológicos de la región. Estas comprenden areniscas con diversos grados de silicificación y composición mineralógica, basaltos, calizas y limolitas. Estas rocas provienen de diferentes formaciones geológicas localizadas dentro de la cuenca del río Paraná y a lo largo de la cuenca del río Uruguay.

Se seleccionaron como variables controladas en los experimentos de uso: materia prima, cinemática, material trabajado, estado del material trabajado, ángulo de trabajo, ángulo del filo, morfología del filo y tiempo de uso. Los resultados obtenidos permitieron identificar y caracterizar los rastros de uso generados a partir del trabajo de diversos materiales con diferentes cinemáticas en estas materias primas poco exploradas desde la perspectiva del análisis funcional. Éstos conforman un marco de referencia sólido para el posterior estudio de los artefactos provenientes de los contextos arqueológicos.
\end{abstract}

Palabras clave: Entre Ríos; materias primas líticas; experimentación; análisis funcional; rastros de uso.

\begin{abstract}
This paper presents the results of the experimental use-wear analysis applied to lithic raw materials from different outcrops of Entre Ríos province. These include sandstones with different degrees of silicification and mineralogical composition, basalts, limestones and siltstones. These rocks come from different geological formations, which are located within the Paraná river basin and along the Uruguay River basin.

The variables controlled in the usewear experiments were: raw material, kinematics, material worked, state of the material worked, angle of work, edge angle, edge morphology and time of use.

The results obtained from the experimental program allowed to identify and characterize the usewear traces generated from the work of different materials with different kinematics in these raw materials which have been little explored from the perspective of use-wear analysis. They form a solid frame of reference for the subsequent study of artifacts from archaeological contexts.
\end{abstract}

Keywords: Entre Ríos province; raw materials; functional analysis; experimentation; use wear.

La disponibilidad de trabajos sistemáticos sobre tecnología lítica en el sur del nordeste argentino ha ido en aumento durante los últimos años a partir de la profundización de las investigaciones en el área. El foco principal de estos trabajos se centró en una amplia variedad de temáticas como: la producción de artefactos y estrategias implementadas en la manufactura (Hocsman, 1999; Buc y Silvestre, 2006; Castro, 2013), el análisis y delimitación de diferentes secuencias de talla (Apolinaire, 2017) o el estudio de las fuentes primarias y secundarias de roca (Bonomo y Blasi, 2010, Loponte et al., 2010; Apolinaire, 2018; Apolinaire et al., 2019). Sin embargo, los enfoques funcionales han sido hasta el momento muy poco explorados a pesar que permiten abordar un aspecto tan importante de la tecnología como las prácticas implicadas en el uso de los artefactos líticos. 
Los procesos de producción y uso de los instrumentos líticos están condicionados tanto por factores ambientales como factores de índole social, los cuales implican elecciones y decisiones que encuentran su correlato material en aspectos concretos tales como la utilización diferencial de materias primas líticas, las técnicas de manufactura y uso de los instrumentos, sus diseños, la existencia o no de reactivación y/o reutilización, los modos de descarte, entre otros (véase Alvarez, 2003, 2004; De Angelis, 2012; Leipus, 2006, 2014; Leipus y Mansur, 2007; Pal, 2012 y bibliografía allí citada). El análisis funcional de base microscópica es una herramienta que permite arribar a una definición de estos aspectos mediante la caracterización de los rastros microscópicos que se desarrollan durante el uso sobre los filos que se encuentran en contacto con diferentes materiales (i. $e$. piel, hueso, madera). Su aplicación brinda información directa acerca de la cinemática del instrumento y del material trabajado, pero también sobre otros aspectos tales como la selección de una u otra materia, la vida útil de un artefacto o filo, la intensidad y modalidad de uso (reactivaciones, enmangamiento, etc). Por otro lado, permite caracterizar alteraciones microscópicas tecnológicas y postdepositacionales, las cuales resultan de suma importancia a la hora de analizar materiales provenientes de contextos arqueológicos. Dentro de este marco, el análisis funcional de base microscópica permite inferir actividades relacionadas con el contexto de uso y deposición (véase Mansur, 1999; Alvarez, 2003, 2004; De Angelis, 2012; Leipus, 2006, 2014; Leipus y Mansur, 2007; Pal, 2012).

En este trabajo se presentan los resultados del programa experimental de rastros de uso aplicado a materias primas procedentes de diversos afloramientos primarios del territorio entrerriano. Estas rocas fueron seleccionadas en función de su presencia en contextos arqueológicos de la región (Bonomo y Blasi, 2010; Loponte et al., 2010; Apolinaire et al., 2019). Su objetivo principal consiste en la caracterización de los rastros de uso experimentales y la descripción e identificación de los mecanismos a través de los cuales fueron desarrollados. Estos estudios posibilitan trazar a futuro los lineamientos de un programa de identificación de rastros de uso sobre materiales arqueológicos elaborados sobre las diversas rocas que se encuentran disponibles en el sur del nordeste argentino.

\section{Experimentación y análisis funcional}

Actualmente es ampliamente aceptado que el análisis funcional de base microscópica se fundamenta en las alteraciones de diversos tipos que se producen durante el uso de un artefacto en su zona activa, es decir en el sector donde el instrumento entra en contacto con el material trabajado. El análisis microscópico de estas alteraciones o rastros de uso brinda información tanto sobre la cinemática implicada en la actividad que los generó así como también sobre el material que se trabajó. Para que la identificación funcional sea válida es necesario conocer cuáles son las características de los rastros de uso que cada tipo de trabajo genera, para lo cual necesariamente se debe realizar experimentación (Castro, 1994; Alvarez, 2003; De Angelis, 2012; Leipus, 2006, 2014; Leipus y Mansur, 2007; Mansur, 1999; Pal, 2013-2015).

A partir del conocimiento de las propiedades físicas y químicas de los materiales (tanto de las materias primas líticas como de cada tipo de material trabajado) y de las acciones puestas en práctica durante el uso, se elabora un modelo sobre las deformaciones de los materiales, sus reacciones y resistencias. Este modelo es el que se pone a prueba en el trabajo experimental y está en constante dialéctica con el análisis de los materiales arqueológicos (Mansur, 1999; Leipus, 2006; Leipus y Mansur, 2007). Los materiales utilizados durante la experimentación corresponden a las categorías de roca que han sido registradas en los contextos arqueológicos de diferentes lugares de la provincia de Entre Ríos. El reconocimiento de estas materias primas y su clasificación se realizó siguiendo los criterios especificados por Apolinaire y colaboradores (2019).

En este sentido, los experimentos deben formar un conjunto organizado, sistemático y coherente en los cuales deben limitarse y controlarse las variables que intervienen durante el uso a los fines de poder ser asociadas de modo preciso con los rastros generados en las zonas activas de los instrumentos.

Se han planteado dos tipos de experimentos en el marco del análisis funcional, los replicativos y los analíticos (Leipus, 2006; Marsh y Ferguson, 2010). Los primeros se basan en la definición de las actividades de uso que se pretenden abordar (i.e. raspado, corte, ahuecado, pulido) y se reproducen experimentalmente para luego observar los resultados generados en términos de rastros en las zonas activas. De este modo, es posible asociar estas evidencias con las actividades realizadas. En este sentido es necesario reproducir estas actividades del modo más fiel posible de acuerdo a las condiciones que se infieren en el pasado y las posibles variaciones introducidas deben ser registradas. En la segunda clase de experimentos, los analíticos, se propone establecer el conjunto de variables que componen una actividad para luego establecer sus interrelaciones y lograr así una definición de los rastros generados en función de un estricto control de las variables implicadas. En este trabajo se ha optado por implementar un enfoque que combina ambas propuestas (Alvarez, 2003; De Angelis, 2012; Leipus, 2006, 2014; Leipus y Mansur, 2007; Mansur, 1999; Pal, 2013-2015 y bibliografía allí citada).

\section{El programa experimental}

\section{Características de las materias primas}

Las fuentes de rocas duras aptas para la manufactura 
de artefactos se encuentran concentradas a lo largo de los dos cursos fluviales principales de la provincia de Entre Ríos (Apolinaire et al., 2019). Sin embargo, existen también pequeños afloramientos discontinuos en sectores específicos de varios tributarios, como en el río Gualeguay. Las muestras de rocas utilizadas en este trabajo proceden de dos sectores: la margen izquierda del río Paraná y sus afluentes entre los departamentos La Paz y Gualeguay, y la margen derecha del río Uruguay y sus afluentes entre los departamentos Concordia y Uruguay (Figura 1) (Apolinaire, 2017; Apolinaire, 2018; Apolinaire et al., 2019). En el primer sector las formaciones geológicas con rocas potencialmente útiles son la Fm. Paraná (areniscas y coquinas) y la Fm. Ituzaingó (areniscas y xilópalos). A lo largo del curso del río Uruguay, por otra parte, se muestrearon las formaciones Serra Geral (basaltos toleíticos masivos y alveolares), Puerto Yeruá (areniscas y calizas silicificadas) y El Palmar (areniscas silicificadas y xilópalos) (Apolinaire, 2017; Apolinaire, 2018; Apolinaire et al., 2019). Las materias primas líticas seleccionadas para este programa experimental comprenden diversas variedades de rocas siliciclásticas que incluyen una amplia variedad de areniscas con diversos grados de silicificación y composición mineralógica, tales como cuarzoarenitas, sublitoarenitas y subarcosas con cementos silíceos, carbonaticos y ferruginosos.

Esta diversidad de rocas, a su vez, fue subdividida en dos grandes categorías: areniscas con cemento silíceo (areniscas silicificadas) y areniscas con cemento carbonático (areniscas carbonáticas), que a su vez fueron clasificadas en función de su formación de origen. La clasificación de las rocas se llevó a cabo siguiendo los lineamientos propuestos por uno de los autores (Apolinaire, 2018, Apolinaire et al., 2019). No se incluyeron en este programa experimental rocas criptocristalinas tales como calcedonias, cuarcitas o xilópalos debido a que ya se cuenta con una importante colección experimental de referencia.

Las muestras de areniscas de la Fm. Ituzaingó presentan generalmente un color marrón amarillento y gran diversidad en cuanto a su calidad para la talla. Comprenden tanto a areniscas silicificadas con cemento silíceo como con cemento carbonático encontrándose ambas clases de manera recurrente en los contextos líticos del suroeste entrerriano. En muestra de mano, las primeras presentan mayor tenacidad, fractura concoidea y un buen grado de cohesión entre los granos; en cambio, las segundas, si bien pueden exhibir una buena consolidación, los planos de fractura son irregulares, se observa mayor friabilidad, superficies más rugosas. Si bien ambas clases de roca resultan fáciles de tallar, en los experimentos de uso las areniscas carbonáticas tienden a desgranarse.

Las areniscas de la Fm. Paraná presentan una elevada tenacidad y clastos pequeños. La talla de estas rocas es dificultosa, pero puede generar fracturas concoideas aptas para la manufactura de instrumentos. Sin embargo, esta materia prima se ha recuperado muy escasamente en los conjuntos líticos del área y generalmente en forma de desechos de talla. Las rocas siliciclasticas de la Fm. Salto Chico consisten fundamentalmente en areniscas sicilificadas con muy buena calidad en función de presentar clastos más pequeños, y cementos silíceos tenaces y de características criptocristalinas (ópalo y calcedonia) (Apolinaire et al. 2019). Estas rocas son fácilmente tallables y presentaron una amplia versatilidad durante los experimentos de uso. Arqueológicamente, estas rocas se encuentran presentes en conjuntos de la cuenca del río Uruguay.

Desde el punto de vista del análisis funcional, las rocas incluidas en este programa experimental pueden
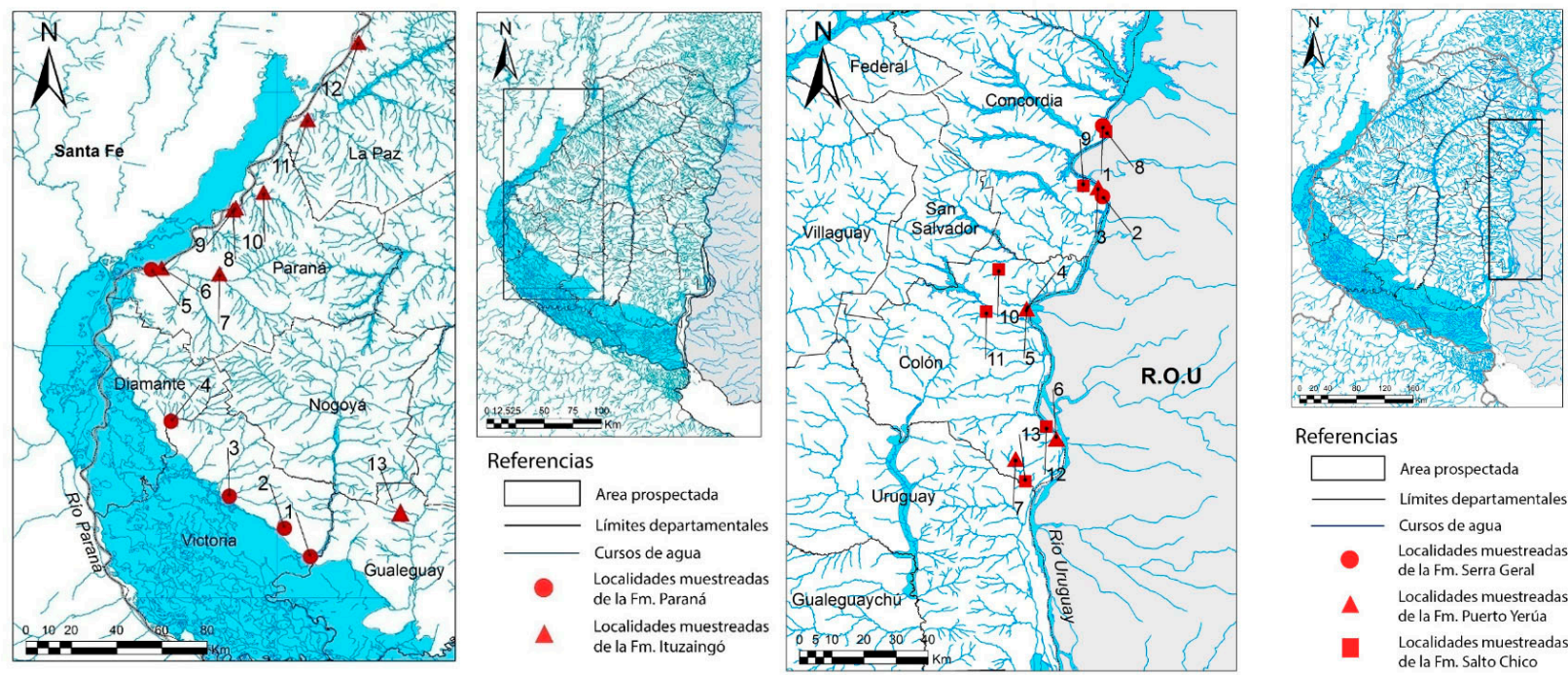

Figura 1. Procedencia de las materias primas utilizadas (modificado de Apolinaire et al. 2019).

Figure 1. Raw material sources (modified from Apolinaire et al. 2019). 
considerarse como materias primas heterogéneas, es decir conformada por cristales de diversos tamaños, cementados por una matriz con diferentes composiciones. A su vez se caracterizan como heterogéneas de grano grueso (i. e. areniscas) en comparación con otras materias primas heterogéneas de grano más fino, tales como los basaltos. Observadas en microscopio óptico de reflexión, las areniscas de la Fm. Ituzaingó y Salto Chico (tanto las que presentan cemento silíceo como carbonático) presentan grandes cristales de cuarzo y escasa matriz de cimentación, características muy similares a las registradas en las ortocuarcitas y metacuarcitas de la región pampeana (Figura 2 A y B) (Leipus, 2006, 2014; Leipus y Mansur, 2007; Pal, 2013-2015). Las areniscas carbonáticas de la Fm Paraná, en cambio, presentan cristales más pequeños que los observados en las areniscas de la Fm Ituzaingó y una mayor cantidad de matriz de cimentación. En las areniscas silicificadas rojizas de la Fm Salto Chico y en los basaltos rojizos de la Fm Serra Geral se registra una situación diferente. En las areniscas también se observan cristales grandes pero abunda el cemento silíceo, asemejándose a las ftanitas pampeanas (Leipus, 2006). Los basaltos presentan, en general, una textura afanítica de manera muy similar a lo observado en otras variedades de basaltos de color negro (Figura 2 C y D) (Leipus, 2006).

\section{Experimentación}

Los artefactos han sido manufacturados a partir de diversos nódulos de las materias primas mencionadas anteriormente. La talla experimental se realizó mediante la extracción de lascas por percusión directa con percutor duro de cuarcita. En el caso de las areniscas silíceas y carbonáticas de la Fm. Ituzaingó, la talla resultó ser una tarea relativamente fácil en comparación con el resto de las materias primas. No se tuvo que implementar demasiada fuerza, debido principalmente a la escasa cohesión entre los granos. Para la talla de las restantes materias primas, si bien resultó ser una tarea efectiva, se tuvo que implementar una mayor cantidad de fuerza.

La selección de las lascas destinadas a su utilización estuvo orientada a incluir la mayor variabilidad posible de productos de la talla en cuanto a su tamaño, ángulos, extensión y morfologías de los filos. De esta manera, el conjunto totaliza 45 filos destinados al procesamiento de diversos materiales bajo diferentes cinemáticas de uso.

Las actividades implicadas en este programa, es decir las formas en las cuales los artefactos líticos se emplearon comprenden una categoría compleja factible de ser subdividida en diferentes variables (i.e. posición del filo, aplicación de la fuerza, ángulo de trabajo). Hasta el momento, en este programa experimental se han realizado solo dos tipos de acciones: transversales (raspar, alisar y cepillar) y longitudinales (aserrar y cortar). Los ángulos de trabajo utilizados influyen significativamente en la ubicación y localización de los rastros de uso. Las acciones transversales se ha trabajado con ángulos comprendidos entre $30^{\circ}$ y $90^{\circ}$ y en las acciones longitudinales con ángulos de trabajo entre $30^{\circ}-35^{\circ}$ y $85^{\circ}-90^{\circ}$. Otra variable crucial es el tiempo de trabajo. A los fines de brindar un buen control de éste, se trabajó en lapsos de $5^{\prime}, 10^{\prime}, 15^{\prime}, 30^{\prime} 60^{\prime}$ y $90^{\prime}$, con la finalidad de registrar los intervalos de desarrollo de los rastros, en particular de los micropulidos.

La morfología de los filos a utilizarse es otra de las variables que influyen de modo importante en la conformación de los rastros. Se seleccionaron filos de diversas morfologías: rectos, convexos, cóncavos e irregulares. Los ángulos de los filos se vinculan a la localización y situación de los rastros de uso y también en la efectividad del trabajo. En el presente programa experimental se han empleado en las acciones transversales filos con ángulos entre 40 a $85^{\circ}$ y en las acciones longitudinales filos con ángulos entre $20^{\circ}$ a $45^{\circ}$.
Figura 2. Características de las rocas en base al análisis funcional de base microscópica, filos sin uso. A) Arenisca silicificada Fm Ituzaingó (cemento silíceo) 200 X. B) Arenisca silicificada Fm Ituzaingó (cemento carbonático ) 200 X. C) Arenisca silicificada Fm Salto Chico 200 X. D) Basalto Fm Serra Geral 200 X

Figure 2. Rock characteristics according to microscopic functional analysis, edges without use. A) Silicified sandstone Fm. Ituzaingo (siliceous cement) 200 $X$. B) Silicified sandstone Fm. Ituzaingo (carbonatic cement) 200 X. C) Silicified sandstone Fm. Salto Chico $200 X^{\prime}$. D) Basalt Fm Serra Geral 200 X
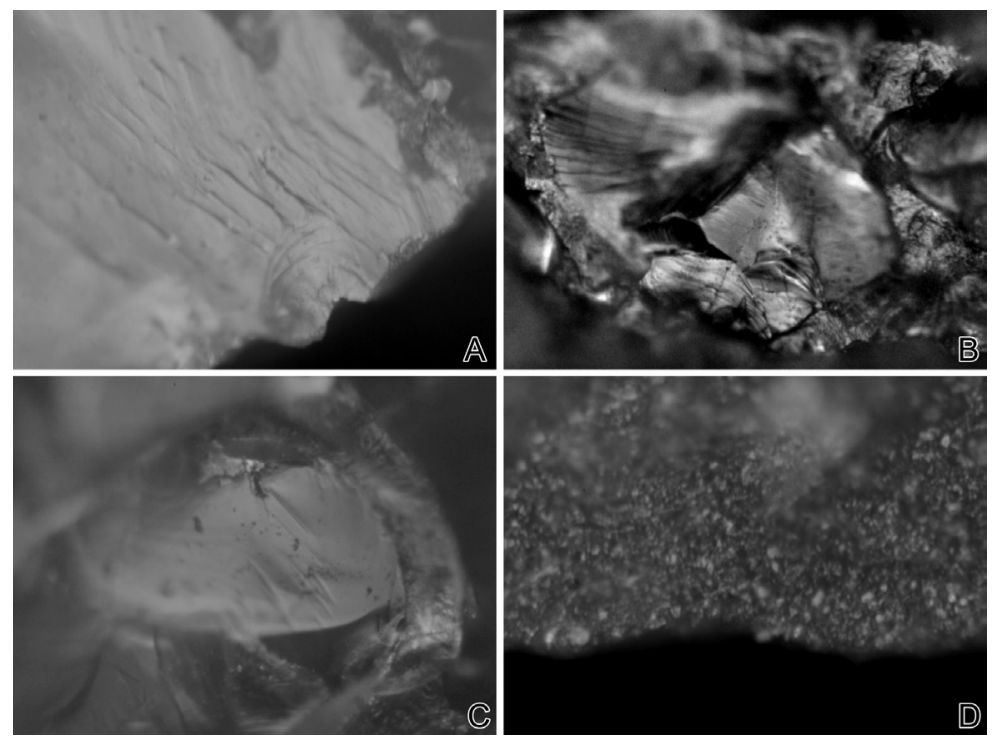
El tipo de sustancia con la cual entra en contacto el filo utilizado influye de manera considerable en el tipo de rastros que se generan con el uso, especialmente, en el tipo y grado de desarrollo de los micropulidos. La cualidad más importante de la sustancia trabajada que se relaciona con los rastros de utilización, es la dureza. Otro factor a considerar es el grado de humedad, ya que influye de manera muy sensible en algunos atributos de los micropulidos: velocidad de desarrollo, brillo, microtopografía, entre otros.

En el presente programa experimental se han incluido maderas de durezas diferentes que se encuentran naturalmente presentes en el territorio entrerriano como es el caso del tala (Celtis tala) y del ceibo (Erithrina crista galli), tanto en estado fresco como seco. Dentro del grupo de las sustancias vegetales no leñosas, se han trabajado diferentes tipos de gramíneas, principalmente hojas y tallos, en acciones de corte. Las sustancias animales procesadas incluyen huesos de vaca (BosTaurus) y pieles de oveja (Ovis aries).

Posteriormente a la realización de la experimentación las piezas utilizadas fueron lavadas con agua y jabón neutro y durante las observaciones microscópicas la limpieza se mantuvo empleando alcohol etílico. Los instrumentos ópticos utilizados fueron una lupa binocular Iroscope YZ-6 con aumentos entre 60 X y 100 X y un microscopio Nikon Epihot 200 con campo claro y oscuro y magnificación de 50 a 500 X, priorizándose las observaciones a 200 X.

\section{Resultados del programa experimental}

Los filos de los artefactos de casi todas las materias primas comprendidas en este programa experimental han presentado rastros diagnósticos en lapsos comprendidos entre los $20^{\prime}$ y los $60^{\prime}$ de uso. Solo en los artefactos elaborados sobre las rocas procedentes de la Fm. Paraná no se observaron rastros diagnósticos a pesar de haber sido utilizados, en algunos casos hasta por 90' (Tabla 1).

\section{Rastros generados por el trabajo en madera}

El desprendimiento de microlascas en los filos usados sobre madera, aunque no fue muy intenso, se hizo más evidente en los filos de areniscas carbonáticas que en las restantes materias primas. Esto se observó cuando se trabajó madera dura y en estado seco, en comparación con maderas más blandas y húmedas. En cuanto al redondeamiento de la arista del filo, se registró una ligera modificación a partir de los 30' de uso, que se hizo más intensa a medida que aumentó el tiempo de utilización (60') y varió según su dureza y resistencia en todas las materias primas. El redondeamiento se desarrolló más rápidamente en trabajos transversales y con ángulos de trabajo más abiertos (i.e. raspar) comparativamente con los longitudinales.

El micropulido se observa muy brillante, con una superficie lisa y regular con ligeras ondulaciones sobre las partes más elevadas de la microtopografía. Cuando se encuentra bien desarrollado, afecta tanto las zonas altas como las deprimidas y toda la superficie adopta un aspecto ondulado y se va haciendo más abierto hacia el interior de la cara, aunque sobre el borde de la arista se observa como una banda espesa. La presencia de humedad es un factor importante en su formación: en los filos usados sobre madera fresca se desarrolló más rápidamente que sobre madera seca. Las estrías son abundantes y se ubican siguiendo la dirección del movimiento realizado.

Los micropulidos comienzan a formarse luego de trabajar madera durante $10^{\prime}$ y $15^{\prime}$ y cuando los filos fueron usados durante períodos de tiempo más prolongados (entre 30' y $60^{\prime}$ ) su presencia se hizo diagnóstica. Es brillante a lo largo de todo el filo y luego de 60' de uso adquiere una apariencia fluída, es espeso, con superficie lisa y regular. Es

Figura 3. Rastros de uso desarrollados por el trabajo sobre madera fresca. A) Arenisca silicificada ( $F m$ Ituzaingó cemento silíceo) Trabajo longitudinal, 30' de uso 200 X. B) Arenisca silicificada (Fm Ituzaingó cemento carbonático) Trabajo transversal, 30' de uso 200 X. C) Arenisca silicificada (Fm Salto Chico) Trabajo transversal, 30' de uso 200 X. D) Basalto (Fm Serra Geral) Trabajo longitudinal, 30' de uso 200 X

Figure 3. Use-marks developed through work over fresh wood

A) Silicified sandstone Fm. Ituzaingo (siliceous cement). Longitudinal work during 30 minutes, 200 $X$. B) Silicified sandstone Fm. Ituzaingo (carbonatic cement). Transversal work during 30 minutes, 200 X. C) Silicified sandstone Fm. Salto Chico. Transversal work during 30 minutes, 200 X. D) Basalt Fm Serra Geral. 30 minutes of use, $200 X$
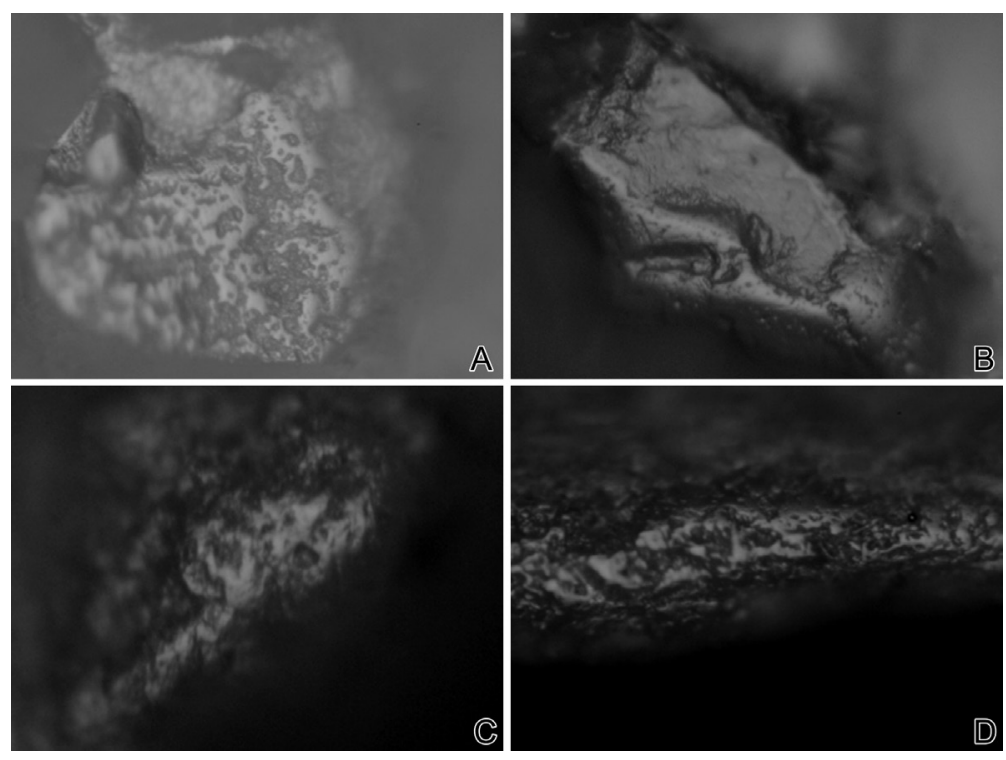
Tabla 1. No: número de pieza; M: materia prima; MF: morfología del filo; $A F$ : ángulo del filo; Cl: cinemática; MT: material trabajado; E: estado del material; TU: tiempo de uso. ASCC: arenisca silificada cemento carbonático; ASCS: arenisca silicificada cemento silíceo; AFP: arenisca Formación Paraná; CS: caliza silicificada; BAS: basalto. CV: convexo; R: recto; I: irregular. T: transversal; L: longitudinal. P: piel; H: hueso; MT: madera de tala; MC: madera de ceibo; G: gramíneas. F: fresco; S: seco.

Table 1. No: artifact number; M: raw material; MF: edge morphology; AF: edge angle; Cl: movement; MT: worked material; E: material status; TU: use time. ACC: carbonatic sandstone; ASCS: silicified sandstone; AFP: Paraná Formation sandstone; CS: silicified limestone; BAS: basalt. CV: convex; R: straight; I: irregular. T: transversal; L: longitudinal. P: fur; H: bone; MT: tala wood; MC: ceibo wood; G: grasses. : fresh; S: dry.

\begin{tabular}{|c|c|c|c|c|c|c|c|}
\hline $\mathrm{N}^{\circ}$ & $\begin{array}{c}\text { Materia } \\
\text { Prima }\end{array}$ & $\begin{array}{l}\text { Morfología del } \\
\text { Filo }\end{array}$ & $\begin{array}{l}\text { Angulo del } \\
\text { Filo }\end{array}$ & Cinemática & \multicolumn{2}{|c|}{$\begin{array}{l}\text { Material } \\
\text { Trabajado }\end{array}$} & $\begin{array}{c}\text { Tiempo de } \\
\text { uso }\end{array}$ \\
\hline 1 & ASCC & $\mathrm{CV}$ & 45 & $T$ & $P$ & $S$ & $45^{\prime}$ \\
\hline 2 & ASCC & $\mathrm{CV}$ & 40 & $T$ & $\mathrm{H}$ & $\mathrm{F}$ & $20^{\prime}$ \\
\hline 3 & ASCC & $\mathrm{CV}$ & 65 & $T$ & MT & $\mathrm{F}$ & $30^{\prime} 60^{\prime}$ \\
\hline 4 & ASCC & $\mathrm{R}$ & 40 & L & $\mathrm{G}$ & $\mathrm{F}$ & $30^{\prime} 60^{\prime}$ \\
\hline 5 & ASCC & $\mathrm{CV}$ & 65 & $T$ & MC & $\mathrm{F}$ & $30^{\prime} 60^{\prime}$ \\
\hline 6 & ASCC & $\mathrm{CV}$ & 20 & $\mathrm{~L}$ & $\mathrm{H}$ & $\mathrm{F}$ & $5^{\prime}$ \\
\hline 7 & ASCC & $\mathrm{R}$ & 45 & $\mathrm{~L}$ & MT & $\mathrm{F}$ & $30^{\prime}$ \\
\hline 8 & ASCC & $\mathrm{CV}$ & 45 & $\mathrm{~T}$ & $P$ & $S$ & $30^{\prime}$ \\
\hline 9 & ASCC & $\mathrm{R}$ & 45 & $\mathrm{~L}$ & G & $\mathrm{F}$ & $15^{\prime}$ \\
\hline 10 & ASCS & $R$ & 80 & $T$ & MC & $\mathrm{F}$ & $45^{\prime}$ \\
\hline 11 & ASCS & $\mathrm{R}$ & 45 & $L$ & $\mathrm{H}$ & $\mathrm{F}$ & $30^{\prime}$ \\
\hline 12 & ASCS & $\mathrm{R}$ & 65 & $T$ & $P$ & $S$ & $30^{\prime} 45^{\prime}$ \\
\hline 13 & ASCS & $\mathrm{R}$ & 40 & $L$ & MC & $\mathrm{F}$ & $30^{\prime}$ \\
\hline 14 & ASCS & $\mathrm{CV}$ & 45 & $L$ & G & $\mathrm{F}$ & $30^{\prime} 60^{\prime}$ \\
\hline 15 & ASCS & $\mathrm{R}$ & 45 & $\mathrm{~L}$ & MT & $\mathrm{F}$ & $10^{\prime} 30^{\prime}$ \\
\hline 16 & ASCS & $\mathrm{CV}$ & 60 & $T$ & $\mathrm{H}$ & $\mathrm{F}$ & $5^{\prime} 15^{\prime}$ \\
\hline 17 & ASCS & $\mathrm{R}$ & 40 & $L$ & G & $\mathrm{F}$ & $15^{\prime}$ \\
\hline \multirow{3}{*}{18} & \multirow{3}{*}{ AFP } & $\mathrm{R}$ & 50 & $T$ & $\mathrm{H}$ & $\mathrm{F}$ & $20^{\prime}$ \\
\hline & & $I$ & 45 & $T$ & $\mathrm{P}$ & $\mathrm{S}$ & $45^{\prime}$ \\
\hline & & $\mathrm{CV}$ & 50 & $\mathrm{~T}$ & $\mathrm{H}$ & $\mathrm{F}$ & $30^{\prime}$ \\
\hline 19 & AFP & $\mathrm{R}$ & 20 & $\mathrm{~L}$ & $\mathrm{H}$ & $\mathrm{F}$ & $10^{\prime}$ \\
\hline \multirow[b]{2}{*}{20} & \multirow[b]{2}{*}{ AFP } & $\mathrm{R}$ & 25 & $\mathrm{~L}$ & MC & $\mathrm{F}$ & $90^{\prime}$ \\
\hline & & $R$ & 30 & L & $\mathrm{MC}$ & $\mathrm{F}$ & $30^{\prime}$ \\
\hline 21 & AFP & $\mathrm{CV}$ & 40 & $\mathrm{~L}$ & G & $\mathrm{F}$ & $30^{\prime} 60^{\prime}$ \\
\hline 22 & AFP & $\mathrm{R}$ & 55 & $T$ & $\mathrm{H}$ & $\mathrm{F}$ & $20^{\prime}$ \\
\hline 23 & AFP & I & 40 & L & G & $\mathrm{F}$ & $15^{\prime}$ \\
\hline 24 & AFP & $\mathrm{CV}$ & 55 & $T$ & MT & $\mathrm{F}$ & $30^{\prime} 60^{\prime}$ \\
\hline 25 & AFP & $\mathrm{CV}$ & 45 & L & MT & $\mathrm{F}$ & $45^{\prime} 60^{\prime}$ \\
\hline 26 & CS & $I$ & 40 & $T$ & $P$ & $S$ & $30^{\prime}$ \\
\hline 27 & CS & $\mathrm{R}$ & 25 & $L$ & $\mathrm{H}$ & $\mathrm{F}$ & $20^{\prime}$ \\
\hline 28 & CS & $R$ & 45 & $T$ & MC & $\mathrm{F}$ & $30^{\prime}$ \\
\hline 29 & CS & $\mathrm{CV}$ & 45 & $\mathrm{~L}$ & G & $\mathrm{F}$ & $30^{\prime} 45^{\prime}$ \\
\hline 30 & CS & $R$ & 45 & $\mathrm{~L}$ & MT & $\mathrm{F}$ & $15^{\prime} 30^{\prime}$ \\
\hline 31 & CS & $\mathrm{CV}$ & 55 & $T$ & $P$ & $S$ & $30^{\prime} 45$ \\
\hline 32 & CS & $\mathrm{CV}$ & 45 & $\mathrm{~L}$ & $\mathrm{H}$ & $\mathrm{F}$ & $10^{\prime} 15^{\prime}$ \\
\hline 33 & BAS & $\mathrm{CV}$ & 85 & $T$ & $P$ & $S$ & $30^{\prime} 45^{\prime}$ \\
\hline 24 & BAS & $R$ & 85 & $T$ & $\mathrm{H}$ & $\mathrm{F}$ & $20^{\prime}$ \\
\hline 35 & BAS & $R$ & 55 & $\mathrm{~T}$ & $\mathrm{H}$ & $\mathrm{F}$ & $20^{\prime}$ \\
\hline 36 & BAS & $\mathrm{CV}$ & 20 & $\mathrm{~L}$ & $\mathrm{MC}$ & $\mathrm{F}$ & $30^{\prime} 60^{\prime}$ \\
\hline \multirow{2}{*}{37} & \multirow{2}{*}{ BAS } & I & 45 & $\mathrm{~T}$ & MC & $\mathrm{F}$ & $30^{\prime} 60^{\prime}$ \\
\hline & & $\mathrm{CV}$ & 55 & $T$ & $M C$ & $\mathrm{~F}$ & $60^{\prime}$ \\
\hline 38 & BAS & CV & 85 & $T$ & $P$ & $S$ & $45^{\prime}$ \\
\hline 39 & BAS & $\mathrm{CV}$ & 45 & $\mathrm{~L}$ & G & $\mathrm{F}$ & $30^{\prime} 60^{\prime}$ \\
\hline 40 & BAS & $\mathrm{R}$ & 60 & $T$ & MT & $\mathrm{F}$ & '10' $30^{\prime}$ \\
\hline 41 & BAS & $\mathrm{CV}$ & 40 & L & $\mathrm{H}$ & $F$ & $5^{\prime} 15^{\prime}$ \\
\hline
\end{tabular}


Figura 4. Rastros de uso desarrollados por el trabajo sobre hueso fresco. A) Arenisca silicificada (Fm Ituzaingó cemento silíceo) Trabajo longitudinal, 30' de uso 200 X . B) Arenisca silicificada (Fm Ituzaingó cemento carbonático) Trabajo transversal, 20' de uso 200 X. C) Arenisca silicificada (Fm Salto chico) Trabajo longitudinal, 20' de uso 200 X. D) Basalto (Fm Serra Geral) Trabajo transversal, $20^{\prime}$ de uso $200 \mathrm{X}$

Figure 4. Use-marks developed through work over fresh bone. A) Silicified sandstone Fm. Ituzaingo (siliceous cement). Longitudinal work during 30 minutes, 200 X. B) Silicified sandstone Fm. Ituzaingo (carbonatic cement). Transversal work during 20 minutes, $200 \mathrm{X}$. C) Silicified sandstone Fm. Salto Chico. Transversal work during 20 minutes, $200 X$. D) Basalt Fm Serra Geral. Transversal work during 20 minutes of use, $200 X$
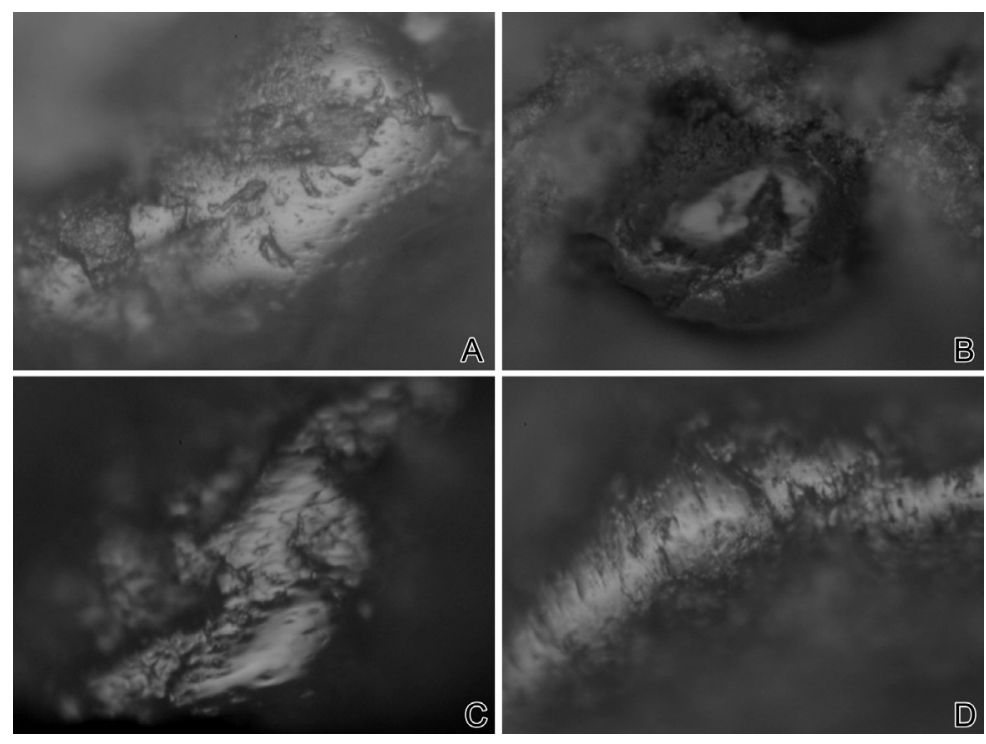

más fácilmente reconocible, cuando se presentan estrías asociadas (colmatadas y no colmatadas), micropoceado o surcos lineales que se orientan en el sentido de la cinemática (Figura 3) (Álvarez, 2003; De Angelis, 2012; Leipus, 2006, 2014; Leipus y Mansur, 2007; Mansur, 1999; Pal, 2013-2015).

\section{Rastros generados por el trabajo en hueso}

El trabajo de hueso produce un intenso embotamiento y deterioro de los filos, que se desarrolla a los pocos minutos de trabajo $\left(5^{\prime}-10^{\prime}\right)$. En los filos experimentales de todas las materias primas los rastros se han observado bien desarrollados y en grado diagnóstico a partir de los $20^{\prime}$ de utilización. En las areniscas carbonáticas se produjo un gran desprendimiento de microlascas a partir de los $5^{\prime}$ de uso, estabilizándose el filo alrededor de los 15'. En todas las materias primas se formaron diferentes tipos de estrías microscópicas: cortas, anchas o estrechas, y no muy profundas y pequeños surcos alineados de forma irregular. Se observan microlascados, los cuales se suelen pulimentar y redondear en grado intenso. Las estrías y rastros de abrasión son frecuentes, pero muchas veces quedan solapados por la gran cantidad de microlascados. El micropulido se forma rápidamente en comparación con otros tipos de rasgos, pero raramente se encuentra ampliamente desarrollado (Álvarez, 2003; De Angelis, 2012; Leipus, 2006, 2014; Leipus y Mansur, 2007; Mansur, 1999; Pal, 2013-2015).

Cuando se comienza a trabajar el hueso se forma un micropulido en estado indiferenciado, el cual de manera rápida se transforma en diagnóstico. En los artefactos experimentales de todas las materias primas se ha observado algún grado de diferenciación luego de los $5^{\prime}$ y $10^{\prime}$ de utilización y con un grado de desarrollo característico del mismo a partir de 20' de uso. Se observa principalmente sobre las zonas salientes del filo, donde el contacto con el material trabajado es más intenso, para extenderse luego hacia el interior de la cara del filo. Se localiza en las zonas altas de la microtopografía. Las zonas del filo con micropulido presentan superficies muy lisas, brillantes, ligeramente onduladas; las zonas no pulidas se presentan como depresiones mate. En general, es poco extenso y generalmente no ocupa la totalidad del filo, solamente ciertas partes como aristas e irregularidades de la superficie.Se observan ciertos rasgos microscópicos como depresiones pequeñas de la microtopografía no colmatadas, estrías de diferentes formas y estriaciones profundas e irregulares con contorno irregular que se orientan en sentido del movimiento.En el micropulido se observan unas finas resquebrajaduras típicas del trabajo de hueso (craquelé) y suelen orientarse en forma perpendicular al movimiento de uso, siendo, anchas y profundas, y en ciertas ocasiones sin colmatar (Figura 4).

\section{Rastros generados por el trabajo en piel}

El rastro más común que se desarrolla es el redondeamiento pronunciado de la arista del filo, el cual es más intenso cuando se ha trabajado piel en estado seco, acompañado de una gran cantidad de estrías de diversos tipos que se localizan siguiendo la dirección del movimiento. El micropulido de piel seca o estacionada es de formación muy lenta. En las piezas experimentales se desarrolló entre los 30' y 45' de uso. Al pasar de un estadio indiferenciado, se vuelve extremadamente rugoso. La superficie del micropulido se presenta con numerosas depresiones pequeñas hemiesféricas, no pulidas. El micropulido es poco brillante, prácticamente mate y su extensión sobre el filo es amplia, dependiendo del ángulo de trabajo. El contacto de los cristales con la matriz se produce de manera brusca ya que los cristales no se redondean como en el caso del trabajo sobre madera o vegetales no leñosos (Figura 5) (Alvarez, 2003; De Angelis, 2012; Leipus, 2006, 2014; Leipus y Mansur, 2007; Mansur, 1999; Pal, 2013-2015).

\section{Rastros generados por el trabajo en vegetales no leñosos}



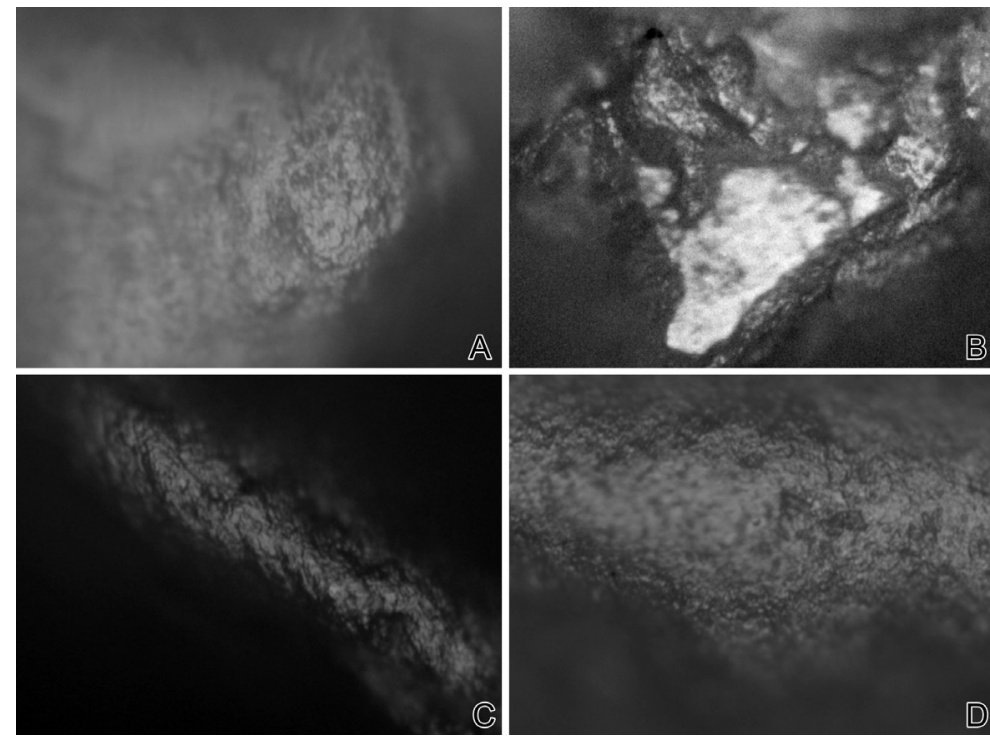

Figura 5. Rastros de uso desarrollados por el trabajo sobre piel seca. A) Arenisca silicificada (Fm Ituzaingó cemento silíceo) Trabajo transversal, 45' de uso 200 X. B) Arenisca silicificada (Fm Ituzaingó cemento carbonático) Trabajo transversal, 45' de uso 200 X. C) Arenisca silicificada (Fm Salto Chico) Trabajo transversal, 30' de uso $200 \mathrm{X}$. D) Basalto (Fm Serra Geral) Trabajo transversal, 45' de uso $200 \mathrm{X}$

Figure 5. Use-marks developed through work over dry leather. A) Silicified sandstone Fm. Ituzaingo (siliceous cement). Transversal work during 45 minutes, 200 X. B) Silicified sandstone Fm. Ituzaingo (carbonatic cement). Transversal work during 45 minutes, $200 X$. C) Silicified sandstone Fm. Salto Chico. Transversal work during 30 minutes, $200 X$. D) Basalt Fm Serra Geral. Transversal work during 45 minutes of use, $200 X$
Durante el trabajo de vegetales no leñosos (i. e. gramíneas) no se desarrollan gran cantidad de estrías ni se forman microlascados. En cuanto a las estrías, se observaron de manera esporádica algunas angostas y superficiales, con colmatación. El micropulido producido se forma muy lentamente en comparación con materiales más duros. En los artefactos experimentales se ha registrado en un estadio diferenciado luego de $30^{\prime}$ de utilización y se observó con sus características ópticas distintivas entre los 30' y los 45' de uso en todas las materias primas. Es muy brillante, siempre forma una cubierta de aspecto fluído, es espeso, con superficie lisa y regular y con algunos rastros característicos como estrías orientadas en la dirección de la utilización. Recubre tanto las partes deprimidas como altas de la microtopografía y los negativos de los microlascados. La presencia de humedad es un factor importante en su formación. Comienza siendo un micropulido moderadamente espeso, regular y muy brillante. Las zonas intersticiales se colmataron con el sucesivo desarrollo del micropulido, que va ocupando las zonas deprimidas de la microtopografía. Se observó un lustre después de pocos minutos de uso y con el incremento en el tiempo de utilización, el micropulido se hizo más luminiscente y diagnóstico.

\section{Consideraciones finales}

Los resultados obtenidos del desarrollo del programa experimental permitieron caracterizar los rastros de uso generados a partir del trabajo de diversos materiales con diferentes cinemáticas, en estas materias primas poco exploradas desde la perspectiva del análisis funcional. Dentro del grupo de las areniscas de la Fm. Ituzaingó se registraron diferencias en cuanto al desarrollo de los rastros de uso. En las areniscas carbonáticas se produjo un esquilamiento del filo más rápido e intenso, sobre todo en el trabajo de materiales duros como el hueso y los micropulidos se desarrollaron de manera lenta. En las areniscas silicificadas, si bien se desprendieron microlascas, el daño no fue tan intenso y los micropulidos se formaron de manera más rápida. Estos resultados son semejantes con los obtenidos en areniscas silicificadas de la Fm. Salto Chico y en los basaltos de la Fm. Serra Geral. En ambos grupos de areniscas los rastros de uso se desarrollaron de manera muy similar a los registrados por uno de nosotros en el programa experimental de cuarcitas procedentes de la Fm. Sierras Bayas y en los diferentes conjuntos líticos arqueológicos de la región pampeana (Flegenheimer y Leipus, 2007; Leipus, 2006, 2014, 2016; Leipus y Mansur, 2007; Pal y Leipus, 2018).

En el caso de las rocas de la Fm Paraná, si bien fueron utilizadas en algunos casos hasta 90', no se registró la presencia de rastros de uso diagnósticos (solo se observaron micropulidos de estadios iniciales). Esto puede deberse a las características estructurales y mineralógicas de las mismas y, en consecuencia, se propone ampliar el tiempo de uso. Los rastros de uso desarrollados en todas las materias primas consideradas en este programa experimental presentan las características propias de cada material trabajado y se correlacionan con las cinemáticas realizadas, en especial los micropulidos, salvo en el caso de las areniscas carbonáticas de la Fm Paraná. Dicho de otra manera, los rastros generados a partir del procesamiento de diversos recursos se desarrollan y presentan características microscópicas semejantes en los diversos tipos de materias primas, resultando equivalentes a los formados en rocas procedentes de otras áreas tales como las ortocuarcitas y metacuarcitas, basaltos, riolitas, calcedonias y ftanitas.

Será necesario ampliar la colección experimental de uso aumentando tanto la cantidad de filos a ser utilizados, 
como así también extender el tiempo de utilización de rocas de la Fm. Paraná. También se prevé la incorporación dentro del programa experimental de otros tipos de materiales a procesar, tales como sustancias minerales colorantes, peces de agua dulce, moluscos y otros materiales.

Finalmente es importante señalar que los resultados presentados aquí conforman un marco de referencia cuyo objetivo último es el análisis de los artefactos líticos provenientes de los sitios arqueológicos del área. A través de estos estudios se podrá discutir con mayor profundidad los variados, y muchas veces contradictorios modelos tecnológicos que han sido planteados para los diversos sectores ecológicos y geográficos que componen la región.

\section{Agradecimientos}

El presente trabajo ha sido desarrollado en el marco del proyecto "Arqueología de ambientes acuáticos del Centro-este argentino" (División Arqueología, FCNyM, UNLP). Nuestro agradecimiento a las Dras. Laura Miotti y Virginia Lynch por facilitar el uso del espacio y del instrumental óptico perteneciente al Laboratorio 17 de la División Arqueología (FCNyM, UNLP) y al Lic. Diego Gobbo (División Arqueología (FCNyM, UNLP) quien estuvo a cargo de la compaginación de las figuras.

\section{Bibliografía}

Álvarez, M. (2003). Organización Tecnológica en el Canal Beagle. El caso de Túnel I (Tierra del Fuego, Argentina). (Tesis doctoral inédita), Universidad de Buenos Aires, Argentina.

Álvarez, M. (2004). Estrategias tecnológicas en los grupos canoeros tempranos del área Fuego-Patagónica. Magallania, 32, 191-208.

Apolinaire, E. (2017). Aprovisionamiento, explotación y circulación de recursos líticos en el Delta Superior del Paraná y las llanuras adyacentes. (Tesis doctoral inédita), Universidad Nacional de La Plata, Argentina.

Apolinaire, E. (2018). Caracterización de los depósitos secundarios de rodados fluviales del río Uruguay inferior: su aplicación en contextos arqueológicos de las llanuras interiores del nordeste argentino. Ciencias Humanas: Boletim do Museu Paraense Emilio Goeldi 13 (2), 433457.

Apolinaire, E., Castiñeira C., \& Bonomo, M. (2019). Nuevos aportes para la delimitación de la base regional de recursos líticos de la provincia de Entre Ríos: relevamiento de afloramientos primarios y caracterización microscópica de rocas siliciclasticas. Arqueología, 25(2),71-22.
Bonomo, M., \& Blasi, A. (2010). Base regional de recursos líticos del Delta del Paraná. Estudio petrográfico de artefactos y afloramientos en el sur de Entre Ríos. Revista Cazadores Recolectores del Cono Sur, 4,17-41.

Buc, N., \& Silvestre, R. (2006). Funcionalidad y complementariedad de los conjuntos líticos y óseos en el humedal del nordeste de la Provincia de Buenos Aires: Anahí, un caso de estudio. Intersecciones en Antropología, 7, 129-146.

Castro, J. (2013). Arqueología del centro de la provincia de Entre Ríos (Argentina). Arqueología, 19(2), 287-304.

De Angelis, H. (2012). Arqueología de la faja central de Tierra del Fuego: una aproximación funcional-espacial. (Tesis doctoral inédita), Universidad Nacional de La Plata, Argentina.

Flegenheimer, N., \& Leipus M. (2007). Trabajar en un espacio reducido, Cerro El Sombrero Abrigo 1. En Actas del XVI Congreso Nacional de Arqueología Argentina (Tomo I, pp. 441-444). Jujuy: FHyCS-UNJu.

Hocsman, S. (1999). Tecnología lítica prehispánica en la cuenca inferior del arroyo Las Conchas (Depto. de Paraná, Pcia. de Entre Ríos): El sitio VU 4 como caso de estudio. (Tesis de grado inédita), Universidad Nacional de Tucumán, Argentina.

Leipus, M. (2006). Análisis de los modos de uso prehispánicos de las materias primas líticas en el Sudeste de la región pampeana: una aproximación funcional. (Tesis doctoral inédita), Universidad Nacional de La Plata, Argentina.

Leipus, M. (2014). El análisis funcional de materias primas heterogéneas y su aplicación a diferentes variedades de cuarcitas de la región Pampeana (Argentina): resultados experimentales y arqueológicos. En Mansur, M. E. Alonso Lima, M., \& Maigrot, Y. (Eds.), Traceology today: Methodological issues in the Old World and the Americas (pp. 43-54). Oxford: BAR International Series.

Leipus, M. (2016). Variabilidad tecnomorfológica y funcional de las raederas en la región Pampeana (Argentina). Revista de Antropología del Museo de Entre Ríos, 2(2), 47-67.

Leipus, M., \& Mansur, M. E. (2007). El análisis funcional de base microscópica aplicado a materiales heterogéneos. Perspectivas metodológicas para el estudio de las cuarcitas de la región Pampeana. En Bayón, C., Pupio, A., González, M. I., Flegenheimer N. y Frere M. (Eds.), Arqueología en las pampas (Tomo I, pp. 179-200). Buenos Aires: Sociedad Argentina de Antropología.

Loponte, D., Tchilinguirian, P., \& Silvestre, R. (2010). 
Caracterización de afloramientos de calizas silicificadas de la provincia de Entre Ríos (Argentina) y su vinculación con los circuitos de abastecimiento prehispánico. Avances y perspectivas en la arqueología del Nordeste, 125-140.

Pal, N., \& Leipus, M. (2018). Modos de producción y uso en la Subregión Pampa Húmeda durante el Holoceno: un enfoque integral de la tecnología lítica de los grupos cazadores-recolectores. En Libro de Resúmenes del I Congreso Argentino de Estudios Líticos en Arqueología (pp. 100-101). Universidad Nacional de Córdoba , Argentina.

Mansur, M. E. (1999). Análisis funcional de material lítico: Problemas de formación y deformación de rastros de uso. En Actas del XII Congreso Nacional de Arqueología Argentina (Tomo I, pp. 355-366). La Plata: Universidad Nacional de La Plata.

Pal, N. (2012). Tendencias temporales en las estrategias de explotación y uso de los materiales líticos recuperados en la cuenca superior del arroyo Tapalqué (partidos de Benito Juárez y Olavarría): una perspectiva desde el análisis funcional. (Tesis doctoral inédita), Universidad Nacional del Centro de la Provincia de Buenos Aires, Argentina.

Pal, N. (2013-2015). Identificación y caracterización de rastros de uso: programa experimental sobre rocas cuarcíticas y ftanita (provincia de Buenos Aires, Argentina). Boletín de Arqueología Experimental, 10, 161-173. 